\title{
DISEASE ACTIVITY PREDICTS WHOLE BODY AND REGIONAL LEAN TISSUE IN RHEUMATOID ARTHRITIS - A CROSS-SECTIONAL STUDY
}

\author{
Claudiu Popescu ${ }^{1,2}$, Violeta Bojinca ${ }^{1,2,3}$, Daniela Opris ${ }^{1,2,3}$, Ruxandra Ionescu ${ }^{1,2,3}$

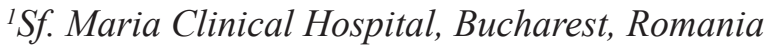 \\ ${ }^{2}$ Department of Internal Medicine and Rheumatology, \\ Carol Davila University of Medicine and Pharmacy, Bucharest, Romania \\ ${ }^{3}$ Research Center of the Pathology and Treatment of Systemic Rheumatic Diseases - RCRD, Bucharest, \\ Romania
}

\begin{abstract}
Aim. The study aims to assess the potential influences of rheumatoid arthritis (RA) and its specific disease measures on lean body composition phenotypes of female patients.

Methods. The study was cross-sectionally designed to include Caucasian postmenopausal female RA patients and age-matched postmenopausal female controls. All the subjects gave written informed consent and the study was approved by the local ethics committee. Each subject underwent in the same day a clinical examination, laboratory tests, whole body dual X-ray absorptiometry (DXA) composition and physical activity estimation using a self-administered questionnaire. Correlations, differences and predictive power were analyzed with appropriate statistical tests.

Results. The study included 107 RA patients and 104 controls. Compared to the normal subjects, who recorded higher levels of physical activity, the RA patients had significantly lower appendicular lean tissue absolute and relative indices and higher prevalence of sarcopenia. The whole body and appendicular lean tissue indices showed significant negative correlations with measures of disease severity (duration, inflammation, quality of life and radiographic progression), independent of age, levels of physical activity, body mass index and smoking.

Conclusions. The measures of disease activity and severity independently predict lean tissue phenotypes in RA patients, behaving as risk factors for sarcopenia and rheumatoid cachexia. The diagnosis of RA in itself is a significant predictive factor of sarcopenia.
\end{abstract}

Keywords: rheumatoid arthritis, dual X-ray absorptiometry, sarcopenia, cachexia

\section{INTRODUCTION}

Rheumatoid arthritis (RA) is a progressive autoimmune disease characterized by chronic inflammation which can lead to permanent joint deformity, disability, distress and socio-economic costs. This disease is also associated with profound modifications of the body composition since the RA-lean tissue interaction leads to sarcopenia and rheumatoid cachexia (1). The main pathogenic factors involved in the RA-associated muscle loss seem to be inadequate physical activity (2) and chronic inflammation-induced catabolism via cytokines such as tumor necrosis factor $\alpha$ (3). The study of these conditions is very relevant since sarcopenia and rheumatoid ca- chexia are associated with low quality of life, chronic fatigue, muscle weakness and adverse outcomes (4). Classical anthropometric measures, such as body mass index (BMI), do not give information on body composition and are not able to discriminate between the proportions of tissue types, which can vary widely within the same BMI ranges. The assessment of body composition in RA patients was mostly done using bioimpedance methods $(5 ; 6)$, which are relatively inexpensive and fast. The use of the more appropriate dual X-ray absorptiometry (DXA) technique for body composition in RA is limited (7-10), especially regarding the whole body and regional muscle mass which are highly correlated with more sensitive estimations methods such

Correspondence address:

Claudiu Popescu, MD, Sf. Maria Clinical Hospital, 37-39 Ion Mihalache boulevard, district 1, Bucharest

E-mail: dr.reumatologie@gmail.com 
as magnetic resonance imaging (11). Most of the studies included both male and female RA patients, although on one hand, RA is more prevalent among women and on the other hand that body composition differs significantly among genders. In this context, the present study aims to evaluate the whole body and regional lean mass in Romanian female RA patients using the DXA technique and to assess the potential influences of RA and its specific disease measures on lean body composition phenotypes of female patients.

\section{METHODS}

\section{Patients and criteria}

The study was cross-sectionally designed to include all the patients admitted to our Rheumatology Department (Research Centre of the Pathology and Treatment of Systemic Rheumatic Diseases - RCRD, Bucharest) in the random order of presentation between May and August 2013, who fulfilled the following criteria: female sex (since RA is more frequent in women and since men have largely different body composition phenotypes than women); Caucasian race; postmenopausal status; 2010 ACR/EULAR classification criteria for RA (12) and more than 6 months disease duration. Age-matched healthy Caucasian post-menopausal female subjects were randomly selected and invited to participate in the study using the records of general practitioners associated with RCRD from the same geographic area. The following exclusion criteria were applied: age under 18 years; pregnancy; weight $>150 \mathrm{~kg}$ (DXA table weight limit); muscular and neuromuscular disease (myositis, muscular dystrophy, myasthenia gravis); current cancer; HIV-AIDS; digestive pathology (gastrectomy, bariatric or intestinal surgery, primary biliary cirrhosis, celiac disease, malabsorption); chronic obstructive lung disease; severe heart failure (New York Heart Association classification $\geq$ 3); endocrine abnormalities (hyperthyroidism, hyperparathyroidism, hypogonadism); moderate-severe chronic kidney disease (glomerular filtration rate $<60 \mathrm{~mL} / \mathrm{min} / 1.73 \mathrm{~m}^{2}$ ); psychiatric eating disorder; therapy with any of the following drugs in the last 6 months: estrogen-replacement therapy, glucocorticoids exceeding $7.5 \mathrm{mg} /$ day oral prednisone equivalent, antipsychotics, anticonvulsants, heparin, orlistat, lorcaserin, exogenous insulin. Each subject gave written informed consent and the study was approved by the local ethics committee. The clinical examination, laboratory tests and DXA body composition analysis were done in the same day for each patient.

\section{Clinical examination}

A clinical interview and a review of the medical history recorded age, smoking status, disease duration, duration of morning stiffness, patient global self assessment of general health and disease activity (visual analog scale), treatment regimes and extraarticular manifestations (rheumatoid nodules, vasculitis, neurologic, pulmonary, cardiac, renal or ophthalmologic involvement). The patients were given a self-administered modified health assessment questionnaire (MHAQ). (13) A single senior author rheumatologist (DO) performed systematical clinical examinations, which focused on anthropometrics and RA variables. Height and weight were measured in upright anatomical position, light clothes, without shoes, using a mechanical scale $(0.1 \mathrm{~kg}$ maximal error) and a stadiometer ( $0.3 \mathrm{~cm}$ maximal error). Obesity and overweight were defined using the World Health Organization (WHO) cutoffs of BMI $\geq 30 \mathrm{~kg} /$ $\mathrm{m}^{2}$ and $25 \mathrm{~kg} / \mathrm{m}^{2}$ respectively. The clinical examination recorded the number of painful and swollen joints (bilateral proximal interphalangeal, metacarpophalangeal, wrist, elbow, shoulder, knee joints) and identified the patients with RA joint deformities of their hands (henceforth designated clinical structural damage): fixed flexion contracture, ulnar deviation, "swan neck", "boutonniere", "Z" thumb, other RA luxations and subluxations, impaired range of motion, arthritis mutilans. A visual analog scale indication of the global disease activity was recorded by the evaluator. A single senior author rheumatologists (VB) identified radiological damage on standard postero-anterior X-ray images of the hands. The Simple Erosion Narrowing Score (SENS) was calculated using the method proposed by van der Heijde et al. (14).

\section{Laboratory measures}

All the included patients underwent morning venipuncture and blood samples were tested with commercial kits for routine blood chemistry and complete blood count, erythrocyte sedimentation rate (ESR; Westergren method), C-reactive protein (CRP; nephelometric method), IgM rheumatoid factor (RF) and anti-citrullinated peptide antibodies (ACPA; enzyme-linked immunosorbent assay). In- 
flammation was classified if either CRP or ESR were above the upper limit of normal $(5 \mathrm{mg} / \mathrm{L}$ and 30 $\mathrm{mm} / \mathrm{h}$ respectively), in the absence of other causes than RA. Using the clinical and laboratory measures, the RA activity was assessed using two composite tools: disease activity score (DAS28; remission $\leq$ 2.6; low disease activity - LDA 2.6-3.2; moderate disease activity - MDA 3.2-5.1; high disease activity - HDA $>5.1)(15)$ and clinical disease activity index (CDAI) (16).

\section{DXA whole body composition}

Body composition was evaluated by whole body less head DXA with a Lexxos C05LX223 densitometer. All the scans were performed by a single certified clinical densitometrist (CP; $0.48 \%$ variation coefficient). Daily calibration and quality control tests were performed according to the manufacturer's recommendations and different regions of interest were manually checked for maximal reliability (17). The patients were required to wear light clothing, without metal or plastic, and were scanned in the morning, after nocturnal fast, micturition and 5-10 minutes of supine rest on the examination table, in the absence of pregnancy and radioactive or radiocontrast investigations in the last week. Data records included whole body and regional (arms, legs) variables such as lean tissue density/mass/area/percent (wbLT D/M/A/P) and whole body adipose tissue mass (wbATM). Appendicular lean mass (ALM) was calculated as the sum of the lean masses of all four members, while the appendicular lean density (ALD) was calculated as the arithmetic mean of the lean tissue densities of the four members. The skeletal muscle mass index (SMI) was calculated in three ways: ALM divided by body mass (SMI1), by square height (SMI2) and by wbLTM (SMI3). The free-fat mass index (FFMI) was calculated in three ways: wbLTM divided by body mass (FFMI1), by square height (FFMI2) and by wbATM (FFMI3). The fat mass index (FMI) was calculated by dividing wbATM to square height. Since the definitions of sarcopenia $(18,19)$ and cachexia $(20)$ include muscle strength, a variable which was not included in the study design, the terms "sarcopenia" and "cachexia" will be used as classification labels for the whole body phenotypes based on low muscle mass and high adipose mass. Defining sarcopenia as SMI2 below 2 standard deviations (SD) of the Rosseta study young female population mean $\left(7.3 \pm 0.9 \mathrm{~kg} / \mathrm{m}^{2}\right)$, or as FFMI2 below the predicted value for Caucasian females (21), resulted in 100\% prevalence of sarcopenia in our sample $(18,22)$. Another strategy was to define sarcopenia as SMI $<20^{\text {th }}$ percentile of the study group $(23,24)$, but unlike the cited authors we did not include male subjects in the study. In the absence of specific Romanian population cutoffs for whole body composition, we used our non-RA group as reference population. Accordingly, sarcopenia was defined by two principles: as SMI2 or FFMI2 < $20^{\text {th }}$ percentile of the non-RA group (corresponding to $3.453 \mathrm{~kg} / \mathrm{m}^{2}$ and $6.874 \mathrm{~kg} / \mathrm{m}^{2}$ respectively); and as T-scores of SMI2 or FFMI2 below 2 SD of the reference group's mean. The T-scores were calculated by subtracting each of the SMI2 or FFMI2 values from the mean of the reference group $\left(4.002 \mathrm{~kg} / \mathrm{m}^{2}\right.$ and $8.075 \mathrm{~kg} / \mathrm{m}^{2}$ respectively) and dividing by the standard deviation of the reference group $\left(0.608 \mathrm{~kg} / \mathrm{m}^{2}\right.$ and $1.357 \mathrm{~kg} / \mathrm{m}^{2}$ respectively). Rheumatoid cachexia was defined as FFMI2 below the $10^{\text {th }}$ percentile and as FMI above the $25^{\text {th }}$ percentile of our reference population (25-27).

\section{Physical activity estimation}

Physical activity was estimated using the selfadministered Global Physical Activity Questionnaire version 2 (GPAQ2), developed by WHO, with a total of 16 questions in 4 activity sections (work, travel, recreation, sedentary behavior) $(28,29)$. The English questionnaire was translated into Romanian by the authors and back-translated by an independent authorized translator. The final version of the Romanian version was pre-tested on a random sample of 15 healthy employees and 15 RA patients from RCRD.

\section{Statistics}

Data distribution normality was assessed using descriptive statistics, normality, stem-and-leaf plots and the Lillefors corrected Kolmogorov-Smirnov test. Qualitative data were expressed as "absolute value (percentage of group)" and were studied using cross-tabs with $\chi^{2}$ or Fisher tests. Non-normally distributed scale data were reported as "median (interval)" or "mean (interquartile range)" and their correlations and differences were assessed using non-parametric tests: bivariate Spearman and partial correlations of 2 scale variables; Mann-Whitney U and Kruskal-Wallis for differences of scale variables in groups with 2 (e.g. smoking) or more categories 
(e.g. RA disease activity). To determine which categories of multi-level nominal variables produced significant $\chi^{2}$ or Mann-Whitney tests, one-way ANOVA was used with post-hoc analysis (Tukey and Bonferroni multiple comparisons). To assess the independent predictive capacity of RA-variables, logistic regression models were created using lean tissue phenotypes (binary logistic regression) or lean tissue scale variables (standard multivariate linear regression) as dependents and RA variables and confounding variables as covariates; the scale variables included in the models were normalized using arithmetic functions (indicated in text). All tests were considered significant if $p<0.05$ and were done us- ing Statistical Package for the Social Sciences v.20 (SPSS Inc., Chicago, USA, 2008) for Windows.

\section{RESULTS}

\section{Comparison of groups}

The study included 107 RA postmenopausal female patients and 104 postmenopausal non-RA female subjects (Table 1$)$. The $49(45.8 \%)$ patients on low-dose glucocorticoids were taking $\leq 7.5 \mathrm{mg} /$ day prednisone for a median period of 9 months (3-15 months). Compared to the normal subjects, who recorded higher levels of physical activity, the RA patients had significantly lower appendicular lean tissue

TABLE 1. General characteristics and comparison of the study groups

\begin{tabular}{|c|c|c|c|}
\hline & non- $R A(n=104)$ & $R A(n=107)$ & $p$ \\
\hline age (years) & $56(48-78)$ & $56(46-76)$ & 0.718 \\
\hline height $(m)$ & $1.58(1.46-1.72)$ & $1.60(1.45-1.75)$ & 0.086 \\
\hline weight $(\mathrm{kg})$ & $72(53-110)$ & $69(43-121)$ & 0.108 \\
\hline BMI $\left(\mathrm{kg} / \mathrm{m}^{2}\right)$ & $28.6(20.1-44.6)$ & $26.7(18.5-42.5)$ & 0.007 \\
\hline wbLTM (kg) & $20.4(10.3-29.9)$ & $19.9(11.7-28.2)$ & 0,835 \\
\hline wbLTD $\left(\mathrm{g} / \mathrm{cm}^{2}\right)$ & $3.93(2.17-5.51)$ & $3.79(2.18-5.35)$ & 0.835 \\
\hline wbLTA $\left(m^{2}\right)$ & $0.53(0.43-0.64)$ & $0.51(0.41-0.67)$ & 0.454 \\
\hline wbLTP (\%) & $35.8(17.7-56.1)$ & $34.4(16.2-57.3)$ & 0.855 \\
\hline $\operatorname{ALD}\left(\mathrm{g} / \mathrm{cm}^{2}\right)$ & $2.99(2.12-4.32)$ & $2.82(2.01-3.79)$ & 0.023 \\
\hline ALM (kg) & $9.74(6.31-15.8)$ & $9.37(5.83-13.1)$ & 0.024 \\
\hline SMI1 & $0.14(0.08-0.21)$ & $0.13(0.08-0.23)$ & 0.688 \\
\hline SMI2 $\left(\mathrm{kg} / \mathrm{m}^{2}\right)$ & $3.94(2.77-5.65)$ & $3.64(2.47-4.77)$ & 0.001 \\
\hline SMI3 & $0.49(0.37-0.88)$ & $0.46(0.36-0.76)$ & 0.005 \\
\hline FFMI1 & $0.29(0.09-0.46)$ & $0.29(0.12-0.48)$ & 0.545 \\
\hline FFMI2 $\left(\mathrm{kg} / \mathrm{m}^{2}\right)$ & 8.16 (4.19-10.7) & $7.81(4.66-10.4)$ & 0.085 \\
\hline FFMI3 & $0.51(0.15-1.27)$ & $0.49(0.16-1.46)$ & 0.707 \\
\hline wbATM & $40.3(19.7-73.2)$ & $39.5(16.7-87.7)$ & 0.330 \\
\hline $\mathrm{FMI}\left(\mathrm{kg} / \mathrm{m}^{2}\right)$ & $16.7(7.31-29.5)$ & $15.5(6.11-30.6)$ & 0.123 \\
\hline smoking $(n)$ & $21(20.2 \%)$ & $18(16.8 \%)$ & 0.298 \\
\hline BMI-obesity (n) & $46(44.2 \%)$ & $25(23.3 \%)$ & 0.007 \\
\hline BMI-overweight (n) & $41(39.4 \%)$ & $48(44.8 \%)$ & 0.491 \\
\hline SMI2-T-sarcopenia & $17(16.9 \%)$ & $39(36.4 \%)$ & 0.004 \\
\hline SMI2-20p-sarcopenia & $21(20.2 \%)$ & $41(38.3 \%)$ & 0.016 \\
\hline FFMI2-T-sarcopenia & $20(19.2 \%)$ & $24(22.4 \%)$ & 0.602 \\
\hline FFMI2-20p-sarcopenia & $17(16.9 \%)$ & $29(27.1 \%)$ & 0.291 \\
\hline TPA (kMET-min/week) & $5.18(0.7-13.7)$ & $4.68(0.5-11.3)$ & 0.041 \\
\hline MTT (min/week) & $13.6(0-154.3)$ & $10.7(0-102.9)$ & 0.045 \\
\hline SB (min/day) & $120(60-480)$ & $300(30-660)$ & 0.033 \\
\hline \multicolumn{4}{|l|}{ levels of physical activity } \\
\hline $\operatorname{low}(n)$ & $7(6.7 \%)$ & $14(13.1 \%)$ & \multirow{3}{*}{0.042} \\
\hline moderate $(n)$ & $34(32.7 \%)$ & $40(37.4 \%)$ & \\
\hline high (n) & $63(60.6 \%)$ & $53(49.5 \%)$ & \\
\hline
\end{tabular}

Notes:

- variables are reported as "median (interval)" and "value (percent of group)";

- $p$ values represent the significance level of the test used to asses differences: Mann-Whitney (scale test variables); $\chi^{2}$ (nominal test variables).

Abbreviations: ALD/M - appendicular lean density/mass; ATM - adipose tissue mass; BMI - body mass index; F/FMI - fat/ free mass index; LT D/M/A/P - lean tissue density/mass/area/percent; MET - metabolic equivalent; MTT - mean travel time; $n$ - number of; RA - rheumatoid arthritis; SB - sedentary behavior; SMI - skeletal muscle index; SMI2/FFMI2-T/20pSP - sarcopenia defined by SMI2/FFMI2 using T-scores or the $20^{\text {th }}$ percentile; TPA - total physical activity; wb - whole body. 
absolute and relative indices and higher prevalence of sarcopenia (22.4-38.3\% according to the definition method).

\section{RA-specific variables}

Compared to the non-sarcopenic RA patients (Table 2), the sarcopenic RA patients, regardless of the definition method, had longer disease duration, higher prevalence of glucocorticoid treatment and rheumatoid cachexia and a more severe and active disease (as measured clinically and radiographically). Table 2 reports only the differences of FFMI-defined sarcopenia by the $20^{\text {th }}$ percentile method, but the other three definitions of sarcopenia behaved the same (data not shown). The sarcopenic patients also displayed lower levels of physical activity and higher levels of sedentary behavior (Table 3). Similarly, compared to the RA patients without cachexia, the cachectic RA patients had longer disease duration and significantly higher radiographic progression (as measured by SENS). Of note, the RA patients with inflammation, clinical structural damage, glucocorticoids and MDA had lower whole body and appendicular lean tissue (Table 4).

The whole body and appendicular lean tissue indices showed significant negative correlations with

TABLE 2. RA-specific variables

\begin{tabular}{|c|c|c|c|c|c|}
\hline \multirow[b]{2}{*}{ variable } & \multirow{2}{*}{$\begin{array}{c}\text { all } \\
(n=107)\end{array}$} & \multicolumn{2}{|c|}{ FFMI2-20p-sarcopenia } & \multicolumn{2}{|c|}{ rheumatoid cachexia } \\
\hline & & no $(n=78)$ & yes $(n=29)$ & no $(n=90)$ & yes $(n=17)$ \\
\hline $\mathrm{ESR}(\mathrm{mm} / \mathrm{h})$ & $30(36)$ & $28(36)$ & $31(41)$ & $28(34)$ & $43(44)$ \\
\hline $\mathrm{CRP}(\mathrm{mg} / \mathrm{L})$ & $6.8(18.8)$ & $6.2(22.9)$ & $9.3(18.6)$ & $6.2(22)$ & $10.8(20)$ \\
\hline inflammation (n) & $70(65.4 \%)$ & $48(61.5 \%)$ & $22(75.9 \%)$ & $56(62.2 \%)$ & $14(82.4 \%)$ \\
\hline RA duration $(y)$ & $10(12)$ & $8(7)$ & $16(21)^{\S}$ & $8(11)$ & $20(23)^{\&}$ \\
\hline stiffness (min) & $30(80)$ & $30(120)$ & $20(60)$ & $30(80)$ & $20(15)$ \\
\hline $\operatorname{CSD}(n)$ & 77 (71.9\%) & $62(79.5 \%)$ & $26(89.7 \%)^{\S}$ & $61(67.8 \%)$ & $15(88.2 \%)$ \\
\hline $\operatorname{EAM}(n)$ & $24(22.4 \%)$ & $24(30.8 \%)$ & $4(13.8 \%)$ & $22(24.4 \%)$ & $2(11.8 \%)$ \\
\hline $\mathrm{RN}(\mathrm{n})$ & $21(19.6 \%)$ & $21(26.9 \%)$ & $4(13.8 \%)$ & $19(21.1 \%)$ & $2(11.8 \%)$ \\
\hline$R F+(n)$ & $93(86.9 \%)$ & $66(84.6 \%)$ & $19(65.5 \%)$ & $77(85.5 \%)$ & $15(88.2 \%)$ \\
\hline $\mathrm{ACPA}+(\mathrm{n})$ & $94(87.8 \%)$ & 67 (85.9\%) & $28(96.5 \%)$ & $78(86.7 \%)$ & $17(100 \%)$ \\
\hline $\operatorname{DMARD}^{*}(\mathrm{n})$ & $96(89.7 \%)$ & $68(87.2 \%)$ & $28(96.5 \%)$ & $80(88.9 \%)$ & $15(88.2 \%)$ \\
\hline biologics $^{\#}(n)$ & $35(32.7 \%)$ & $24(30.8 \%)$ & $11(37.9 \%)$ & $29(32.2 \%)$ & $6(35.3 \%)$ \\
\hline $\mathrm{GC}(\mathrm{n})$ & $49(45.8 \%)$ & $30(38.5 \%)$ & $19(65.5 \%)^{\S}$ & $38(42.2 \%)$ & $11(64.7 \%)$ \\
\hline DAS28 $8_{\text {ESR }}$ & $4.38(1.81)$ & 4.35 (1.99) & $4.51(1.19)$ & $4.32(2)$ & $4.68(0.91)$ \\
\hline DAS28 ${ }_{C R P}$ & $3.94(1.64)$ & $3.87(1.86)$ & $3.98(1.09)$ & $3.86(1.88)$ & $4.06(0.95)$ \\
\hline CDAI & $13.6(11.5)$ & $13.4(12.7)$ & $13.9(6.2)$ & $13(12)$ & $14.7(6.8)$ \\
\hline $\mathrm{HAQ}$ & $0.63(0.75)$ & $0.63(0.75)$ & $1(0.69)^{\S}$ & $0.63(0.88)$ & $0.7(0.6)$ \\
\hline SENS & $22(28)$ & $21(17)$ & $46(40)^{\S}$ & $21(21)$ & $43.5(40)^{8}$ \\
\hline $\mathrm{RC}(\mathrm{n})$ & $17(15.9 \%)$ & $1(1.3 \%)$ & $16(55.2 \%)^{\S}$ & - & - \\
\hline \multicolumn{6}{|c|}{ DAS28 ${ }_{E S R}$ disease activity } \\
\hline remission $(n)$ & $11(10.3 \%)$ & $9(11.5 \%)$ & $2(6.9 \%)$ & $11(12.2 \%)$ & $0(0 \%)$ \\
\hline $\operatorname{low}(n)$ & $13(12.1 \%)$ & $11(14.1 \%)$ & $2(6.9 \%)$ & $13(14.4 \%)$ & $0(0 \%)$ \\
\hline moderate $(n)$ & $54(50.5 \%)$ & $36(46.2 \%)$ & $18(62.1 \%)$ & $41(45.6 \%)$ & $13(76.5 \%)$ \\
\hline high (n) & $29(27.1 \%)$ & $22(28.2 \%)$ & $7(24.1 \%)$ & $25(27.8 \%)$ & $4(23.5 \%)$ \\
\hline \multicolumn{6}{|c|}{ CDAl disease activity\& } \\
\hline remission $(n)^{\ddagger}$ & $6(5.6 \%)$ & $6(7.7 \%)$ & $0(0 \%)$ & $6(6.7 \%)$ & $0(0 \%)$ \\
\hline $\operatorname{low}(n)$ & $25(23.4 \%)$ & $21(26.9 \%)$ & $4(13.8 \%)$ & $25(27.8 \%)$ & $0(0 \%)$ \\
\hline moderate $(n)^{\ddagger}$ & $61(57 \%)$ & $39(50 \%)$ & $22(75.9 \%)$ & $45(50 \%)$ & $16(94.1 \%)$ \\
\hline high (n) & $15(14 \%)$ & $12(15.4 \%)$ & $3(10.3 \%)$ & $14(15.6 \%)$ & 1 (5.9\%) \\
\hline
\end{tabular}

Notes:

* methotrexate, leflunomide, sulfasalazine, hydroxychloroquine, azathioprine, cyclosporine;

\# infliximab, etanercept, adalimumab, golimumab, certolizumab, rituximab, abatacept, tocilizumab;

¥ categories which differed significantly one from another;

- variables are reported as "median (interquartile range)" and "value (percent of group)";

- the test used to asses differences were Mann-Whitney (scale test variables); $\chi^{2}$ (nominal test variables), Kruskal-Wallis for disease activity and ANOVA with post-hoc analysis (CDAl activity) with the following significance levels: $\S p<0.03 ; \& p<0.05$; non-significant if unmarked.

Abbreviations: ACPA - anti-citrullinated protein antibodies; CSD - clinical structural damage; CDAI - clinical disease activity index; CRP - C reactive protein; DAS - disease activity score; DMARD - disease-modifying antirheumatic drugs; EAM - extra-articular manifestations; ESR - erythrocyte sedimentation rate; FFMI2-20p-sarcopenia - sarcopenia defined by FFMI2 using the $20^{\text {th }}$ percentile; GC - glucocorticoids; HAQ - health assessment questionnaire; $\mathrm{n}$ - number (observed value); RA - rheumatoid arthritis; RC - rheumatoid cachexia; RF - rheumatoid factor; RN - rheumatoid nodules; SENS - Simple Erosion Narrowing Score; wb - whole body; y - years. 
TABLE 3. Differences of physical activity among RA phenotypes

\begin{tabular}{|c|c|c|c|c|}
\hline & TPA & MT-TPA & MTT & $S B$ \\
\hline \multicolumn{5}{|c|}{ SMI-sarcopenia - T-score method } \\
\hline no $(n=68)$ & $6.51(5.88)$ & $\begin{array}{c}218 \\
(208)\end{array}$ & $17.2(52.5)$ & $240(240)$ \\
\hline yes $(n=39)$ & $4.08(5.71)$ & $\begin{array}{c}147 \\
(204) \\
\end{array}$ & $8.57(20.7)$ & $360(225)$ \\
\hline $\mathrm{p}$ & 0.011 & 0.020 & 0.020 & 0.020 \\
\hline \multicolumn{5}{|c|}{ SMI-sarcopenia $-20^{\text {th }}$ percentile method } \\
\hline no $(n=66)$ & $6.72(5.88)$ & $\begin{array}{c}216 \\
(210) \\
\end{array}$ & $15.1(52.8)$ & $240(240)$ \\
\hline yes $(n=41)$ & $4.08(5.56)$ & $\begin{array}{c}148 \\
(199)\end{array}$ & $8.67(19.9)$ & $360(210)$ \\
\hline $\mathrm{p}$ & 0.014 & 0.025 & 0.025 & 0.026 \\
\hline \multicolumn{5}{|c|}{ FFMI-sarcopenia - T-score method } \\
\hline no $(n=83)$ & $5.28(6.91)$ & $\begin{array}{c}186 \\
(225) \\
\end{array}$ & $14(40.7)$ & $240(300)$ \\
\hline yes $(n=24)$ & $5.04(5.82)$ & $\begin{array}{c}180 \\
(208) \\
\end{array}$ & $10(26.4)$ & $360(150)$ \\
\hline $\mathrm{p}$ & 0.204 & 0.356 & 0.535 & 0.045 \\
\hline \multicolumn{5}{|c|}{ FFMI-sarcopenia - $20^{\text {th }}$ percentile method } \\
\hline no $(n=78)$ & $5.28(6.91)$ & $\begin{array}{c}186 \\
(225) \\
\end{array}$ & $14.3(40.7)$ & $240(240)$ \\
\hline yes $(n=29)$ & $5.16(5.94)$ & $\begin{array}{c}184 \\
(212) \\
\end{array}$ & $8.57(27.9)$ & $360(180)$ \\
\hline $\mathrm{p}$ & 0.653 & 0.374 & 0.250 & 0.046 \\
\hline \multicolumn{5}{|c|}{ rheumatoid cachexia } \\
\hline no $(n=90)$ & $5.46(6.71)$ & $\begin{array}{c}187 \\
(220) \\
\end{array}$ & $21.4(37.5)$ & $270(225)$ \\
\hline yes $(n=17)$ & $4.68(7.56)$ & $\begin{array}{c}167 \\
(270) \\
\end{array}$ & $12.9(41.3)$ & $360(210)$ \\
\hline $\mathrm{p}$ & 0.135 & 0.429 & 0.135 & 0.042 \\
\hline
\end{tabular}

Notes:

- $p$ values represent the significance level of Mann-Whitney tests;

- units of physical activity indices: TPA [kMET-min/week]; MT-TPA [min/ day]; MTT [min/week]; SB [min/day].

Abbreviations: FFMI - fat-free mass index; MET - metabolic equivalent; MTT - mean travel time per week; MT-TPA - mean time of total physical activity per day; $\mathrm{n}$ - number of; RA - rheumatoid arthritis; SB - sedentary behavior; SMI - skeletal muscle index; TPA - total physical activity per week;

the measures of disease severity (duration, inflammation, quality of life and radiographic progression), independent of age, the levels of physical activity and with the BMI and smoking status (Table 5). In fact, these disease measures were capable to independently predict lean tissue phenotypes in RA patients, behaving as risk factors for sarcopenia and cachexia (Tables 5 and 6). The diagnosis of RA is a significant predictive factor of sarcopenia defined by SMI, but not by FFMI.

\section{DISCUSSION}

\section{Unique study findings}

Our results suggest that RA becomes a significant predictor of the low muscle mass through three inter-
TABLE 4. Significant differences among RA subgroups

\begin{tabular}{|c|c|c|c|}
\hline \multirow[t]{2}{*}{ lean tissue } & \multicolumn{2}{|c|}{ inflammation } & \multirow[t]{2}{*}{$p$} \\
\hline & no $(n=37)$ & yes $(n=70)$ & \\
\hline $\mathrm{wbLTD}\left(\mathrm{g} / \mathrm{cm}^{2}\right)$ & $4.21(1.08)$ & $3.72(1.08)$ & 0.015 \\
\hline wbLTP (\%) & $39.3(10.1)$ & $32.5(13.4)$ & 0.026 \\
\hline $\operatorname{ALD}\left(\mathrm{g} / \mathrm{cm}^{2}\right)$ & $3.12(0.52)$ & $2.73(0.57)$ & 0.003 \\
\hline ALM (kg) & $10.1(2.98)$ & $9.15(2.53)$ & 0.014 \\
\hline SMI1 & $0.15(0.04)$ & $0.12(0.04)$ & 0.007 \\
\hline $\mathrm{SMI} 2\left(\mathrm{~kg} / \mathrm{m}^{2}\right)$ & $3.99(0.92)$ & $3.53(0.89)$ & 0.013 \\
\hline FFMI1 & $0.33(0.09)$ & $0.27(0.11)$ & 0.030 \\
\hline FFMI3 & $0.61(0.26)$ & $0.44(0.31)$ & 0.035 \\
\hline \multirow[t]{3}{*}{ SB (min/day) } & $210(240)$ & $330(195)$ & 0.017 \\
\hline & \multicolumn{2}{|c|}{ clinical structural damage } & \\
\hline & no $(n=30)$ & yes $(n=77)$ & \\
\hline wbLTM (kg) & $21.1(4.38)$ & $18.4(5.83)$ & 0.007 \\
\hline wbLTA $\left(m^{2}\right)$ & $0.55(0.05)$ & $0.51(0.06)$ & 0.001 \\
\hline ALM $(\mathrm{kg})$ & $10.4(1.64)$ & $8.65(2.37)$ & 0.001 \\
\hline $\mathrm{SMI} 2\left(\mathrm{~kg} / \mathrm{m}^{2}\right)$ & $4.01(0.66)$ & $3.49(0.94)$ & 0.001 \\
\hline \multirow[t]{3}{*}{ FFMI2 $\left(\mathrm{kg} / \mathrm{m}^{2}\right)$} & $8.16(1.17)$ & $7.39(1.86)$ & 0.041 \\
\hline & \multicolumn{2}{|c|}{ glucocorticoids } & \\
\hline & no $(n=58)$ & yes $(n=49)$ & \\
\hline $\mathrm{wbLTD}\left(\mathrm{g} / \mathrm{cm}^{2}\right)$ & $4.11(1.08)$ & $3.68(1.14)$ & 0.025 \\
\hline wbLTP (\%) & $36.2(11.1)$ & $32.6(12.1)$ & 0.020 \\
\hline $\operatorname{ALD}\left(\mathrm{g} / \mathrm{cm}^{2}\right)$ & $2.93(0.58)$ & $2.74(0.57)$ & 0.036 \\
\hline SMI1 & $0.15(0.04)$ & $0.12(0.03)$ & 0.013 \\
\hline $\mathrm{SMI}\left(\mathrm{kg} / \mathrm{m}^{2}\right)$ & $3.84(0.78)$ & $3.48(0.97)$ & 0.042 \\
\hline FFMI1 & $0.33(0.09)$ & $0.28(0.11)$ & 0.027 \\
\hline FFMI2 $\left(\mathrm{kg} / \mathrm{m}^{2}\right)$ & $7.99(1.75)$ & $7.08(1.71)$ & 0.035 \\
\hline \multirow[t]{3}{*}{ FFMI3 } & $0.52(0.28)$ & $0.44(0.29)$ & 0.033 \\
\hline & \multicolumn{2}{|c|}{ DAS28 $8_{F S R}$ activity } & \\
\hline & $R(n=11)$ & $\operatorname{MDA}(n=54)$ & \\
\hline wbLTP (\%) & $41.9(16.5)$ & $32.3(12.2)$ & 0.041 \\
\hline SMI1 & $0.16(0.04)$ & $0.13(0.04)$ & 0.040 \\
\hline \multirow[t]{3}{*}{ FFMI1 } & $0.35(0.13)$ & $0.26(0.11)$ & 0.039 \\
\hline & \multicolumn{2}{|c|}{ CDAl activity } & \\
\hline & $R(n=6)$ & $\operatorname{MDA}(n=61)$ & \\
\hline wbLTP (\%) & $48.6(19.2)$ & $32.5(12.6)$ & 0.046 \\
\hline SMI1 & $0.18(0.07)$ & $0.13(0.04)$ & 0.046 \\
\hline FFMI1 & $0.39(0.16)$ & $0.26(0.11)$ & 0.039 \\
\hline
\end{tabular}

Note: reported $\mathrm{p}$ values represent the significance level of Mann-Whitney and Kruskal-Wallis tests.

Abbreviations: ALD/M - appendicular lean density/mass; CDAI - clini$\mathrm{cal} /$ simplified disease activity index; DAS - disease activity score; ESR - erythrocyte sedimentation rate; FFMI - fat-free mass index; LT $\mathrm{D} / \mathrm{M} / \mathrm{A} / \mathrm{P}$ - lean tissue density/mass/area/percent; MDA - moderate disease activity; $\mathrm{R}$ - remission; RA - rheumatoid arthritis; SB - sedentary behavior; SMI - skeletal muscle index; wb- whole body.

twined mechanisms: anatomic joint damage (clinical structural damage and SENS), disease activity (DAS28 and CDAI activity classes) and inflammation (CRP, ESR). The RA patients with anatomical joint damage, high disease activity and chronic inflammation exhibit lower whole body and appendicular lean tissue indices and higher prevalence of sarcopenia and rheumatoid cachexia. These three factors might have a direct effect on muscle mass 
TABLE 5. Significant RA predictors of lean tissue nominal variables

\begin{tabular}{|c|c|c|c|c|c|}
\hline \multicolumn{6}{|c|}{ Correlations of lean tissue and RA variables } \\
\hline lean tissue & RA duration & SENS & $E S R$ & CRP & $H A Q$ \\
\hline wbLTD $\left(\mathrm{g} / \mathrm{cm}^{2}\right)$ & $-0.302^{\#}$ & $-0.355^{\#}$ & ns & ns & ns \\
\hline wbLTM (kg) & $-0.420^{*}$ & $-0.417^{*}$ & ns & ns & ns \\
\hline wbLTP (\%) & $-0.236^{\S}$ & $-0.289^{\S}$ & ns & $-0.239^{\S}$ & ns \\
\hline $\operatorname{ALD}\left(\mathrm{g} / \mathrm{cm}^{2}\right)$ & $-0.409^{*}$ & $-0.462^{*}$ & $-0.292^{\S}$ & $-0.264^{\S}$ & $-0.281^{\S}$ \\
\hline ALM (kg) & $-0.481^{*}$ & $-0.494^{*}$ & ns & ns & $-0.251^{\S}$ \\
\hline SMI1 & $-0.393^{\#}$ & $-0.410^{*}$ & $-0.255^{\S}$ & $-0.294^{\#}$ & $-0.275^{\S}$ \\
\hline $\mathrm{SMI}\left(\mathrm{kg} / \mathrm{m}^{2}\right)$ & $-0.466^{*}$ & $-0.497^{*}$ & ns & ns & $-0.262^{\S}$ \\
\hline FFMI1 & $-0.239^{\S}$ & $-0.276^{\S}$ & ns & $-0.242^{\S}$ & ns \\
\hline FFMI2 $\left(\mathrm{kg} / \mathrm{m}^{2}\right)$ & $-0.383^{\#}$ & $-0.403^{*}$ & ns & ns & ns \\
\hline FFMI3 & $-0.238^{\S}$ & $-0.295^{\#}$ & ns & $-0.235^{\S}$ & ns \\
\hline \multicolumn{6}{|c|}{ Odds ratios of significant predictors of low muscle mass } \\
\hline & \multicolumn{5}{|c|}{ SMI2-sarcopenia } \\
\hline & \multicolumn{2}{|l|}{ T-scores method } & \multicolumn{3}{|c|}{$20^{\text {th }}$ percentile method } \\
\hline diagnosis & \multicolumn{2}{|l|}{$3.571(1.391-6.536)^{\ddagger}$} & \multicolumn{3}{|c|}{$2.728(1.268-5.871)^{\ddagger}$} \\
\hline RA-duration & \multicolumn{2}{|l|}{$1.109(1.045-1.077)^{* *}$} & \multicolumn{3}{|c|}{$1.102(1.040-1.168)^{* *}$} \\
\hline CSD & \multicolumn{2}{|l|}{$1.823(1.201-2.458)^{9}$} & \multicolumn{3}{|c|}{$1.328(1.104-2.269)^{9}$} \\
\hline glucocorticoids & \multicolumn{2}{|l|}{$1.447(1.258-1.676)^{\Uparrow}$} & \multicolumn{3}{|c|}{$2.126(1.115-2.725)^{9}$} \\
\hline $\mathrm{HAQ}$ & \multicolumn{2}{|l|}{$2.015(1.145-5.378)^{\uparrow}$} & \multicolumn{3}{|c|}{$1.837(1.211-4.802)^{9}$} \\
\hline \multirow[t]{3}{*}{ SENS } & \multicolumn{2}{|l|}{$1.051(1.018-1.085)^{\pi}$} & \multicolumn{3}{|c|}{$1.049(1.017-1.082)^{\ddagger}$} \\
\hline & \multicolumn{5}{|l|}{ FFMI2-sarcopenia } \\
\hline & \multicolumn{2}{|l|}{ T-scores method } & \multicolumn{3}{|c|}{$20^{\text {th }}$ percentile method } \\
\hline RA-duration & \multicolumn{2}{|l|}{$1.082(1.026-1.140)^{\ddagger}$} & \multicolumn{3}{|c|}{$1.068(1.017-1.122)^{\ddagger}$} \\
\hline CSD & \multicolumn{2}{|l|}{$1.998(1.345-3.452)^{\pi}$} & \multicolumn{3}{|c|}{$1.678(1.215-3.872)^{\natural}$} \\
\hline glucocorticoids & \multicolumn{2}{|l|}{$1.603(1.059-2.853)^{\pi}$} & \multicolumn{3}{|c|}{$1.127(1.018-1.601)^{\natural}$} \\
\hline \multirow[t]{2}{*}{ SENS } & \multicolumn{2}{|l|}{$1.051(1.021-1.081)^{\ddagger}$} & \multicolumn{3}{|c|}{1.045 (1.017-1.074) } \\
\hline & \multicolumn{5}{|l|}{ rheumatoid cachexia } \\
\hline RA-duration & $1.072(1.015-1.132)^{\S}$ & & & & \\
\hline SENS & $1.042(1.011-1.073)^{\S}$ & & & & \\
\hline $\begin{array}{l}\text { Notes: } \\
\text { - reported correlat } \\
\text { ing status, with the } \\
\text { - reported odds rat } \\
\text { BMI and smoking s } \\
\text { - RA-duration, HAC } \\
\text { "yes"; diagnosis is } \\
\text { Abbreviations: ALD } \\
\text { CRP - C reactive pr } \\
\text { free mass index; H } \\
\text { - non-significant; } \\
\text { Score; wb - whole }\end{array}$ & $\begin{array}{l}\text { ons are two-tailed partial } \\
\text { following significance lev } \\
\text { ( }(95 \% \mathrm{CI}) \text { are from binar } \\
\text { atus, with the following } \\
\text { and SENS are continuol } \\
\text { oded "0" for "non-RA" ar } \\
\text { 'M - appendicular lean } \\
\text { tein; CSD - clinical struc } \\
\text { Q - health assessment } \\
\text { A - rheumatoid arthritis; } \\
\text { ody. }\end{array}$ & $\begin{array}{l}\text { I correlatic } \\
\text { vel: * } p \leq 0 \\
\text { ry logistic } \\
\text { significanc } \\
\text { us; CSD ar } \\
\text { nd "1" for } \\
\text { density/m } \\
\text { ctural dam } \\
\text { questionn } \\
\text {; SMI - sk }\end{array}$ & $\begin{array}{l}\text { ntrolling } \mathrm{fc} \\
\# \mathrm{p} \leq 0.01 \\
\text { ssion mode } \\
\text { el: } * * \mathrm{p} \leq 0 \\
\text { ucocorticoi } \\
\text { BMI - body } \\
\text { ESR - eryth } \\
\text { LT D/M/P - } \\
\text { I muscle in }\end{array}$ & $\begin{array}{l}\text { physical a } \\
0.05 \text {. } \\
\text { ntrolled for } \\
\neq \leq 0.01 ; \text { ๆ } \\
\text { coded " } 0 \text { " } \\
\text { s index; Cl } \\
\text { e sediment } \\
\text { tissue den } \\
\text { ENS - Simr }\end{array}$ & $\begin{array}{l}\text { y, BMI and smok- } \\
\text { physical activity, } \\
\text { "no" and "1" for } \\
\text { fidence interval; } \\
\text { rate; FFMI - fat- } \\
\text { mass/percent; ns } \\
\text { osion Narrowing }\end{array}$ \\
\hline
\end{tabular}

(generalized muscle catabolism in chronic inflammation and periarticular appendicular muscle atrophy) and an indirect influence on body composition (lower levels of physical activity, secondary osteoarthritis). These findings have implications in fundamental research (e.g. the relationship of muscle mass and insulin resistance and metabolic syndrome in RA) and translational clinical potential (e.g. the relationship between the risk of falling and fragility fractures and the whole body or appendicular lean tissue; the effect of targeted treatment on body composition). A prospective study which would observe the evolution of DXA-measured lean tissue under treatment is recommended in order to confirm our results and to document the dynamic extent of the body composition alteration in RA.

\section{Comparing with other studies}

There are few data regarding DXA-estimated whole body and regional lean tissue in women with RA. Generally, recent studies reported no significant difference of absolute whole body lean tissue between RA patients and controls $(7,9,30)$, although there are reports which find the opposite $(31,32)$. In the later studies, the RA patients had lower whole body lean tissue than controls, but the difference was not significant in the study setting, as we also found. 
TABLE 6. Significant RA predictors of lean tissue scale indices

\begin{tabular}{|c|c|c|c|c|c|c|c|}
\hline & independent & $R^{2}$ & B & $S E$ & $p$ & B 95\% Cl & Notes: \\
\hline \multirow{4}{*}{$\begin{array}{l}\frac{\theta}{3} \\
\frac{0}{3}\end{array}$} & disease duration & 0.132 & -0.024 & 0.007 & 0.001 & $-0.038--0.010$ & \multirow{47}{*}{ 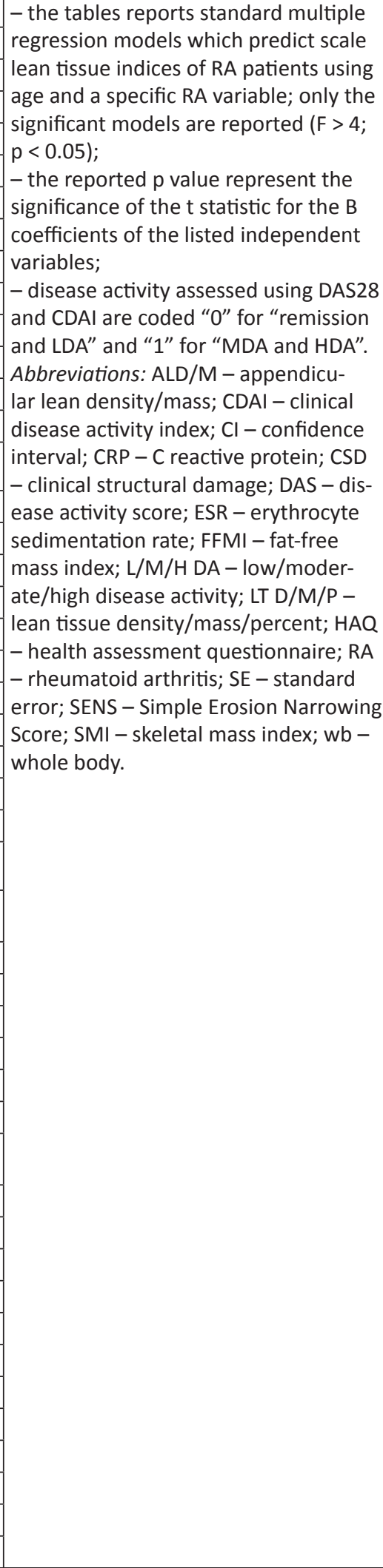 } \\
\hline & SENS & 0.150 & -0.013 & 0.004 & 0.001 & $-0.021--0.006$ & \\
\hline & inflammation & 0.064 & -0.364 & 0.162 & 0.028 & $-0.687--0.040$ & \\
\hline & glucocorticoids & 0.062 & -0.342 & 0.155 & 0.030 & $-0.650--0.034$ & \\
\hline \multirow{3}{*}{$\frac{\sum}{\frac{a}{3}}$} & disease duration & 0.197 & -0.154 & 0.036 & $<0.001$ & $-0.226--0.082$ & \\
\hline & SENS & 0.193 & -0.081 & 0.019 & 0.000 & $-0.119--0.042$ & \\
\hline & CSD & 0.082 & -2.314 & 0.902 & 0.012 & $-4.111--0.517$ & \\
\hline \multirow{4}{*}{$\begin{array}{l}\frac{k}{b} \\
\frac{a}{3}\end{array}$} & disease duration & 0.071 & -0.218 & 0.092 & 0.020 & $-0.401--0.036$ & \\
\hline & SENS & 0.087 & -0.127 & 0.048 & 0.010 & $-0.223--0.032$ & \\
\hline & glucocorticoids & 0.068 & -4.433 & 1.916 & 0.023 & $-8.252--0.615$ & \\
\hline & DAS28 $8_{\mathrm{ESR}}$ activity & 0.062 & -5.072 & 2.299 & 0.030 & $-9.654--0.491$ & \\
\hline \multirow{7}{*}{$\stackrel{\ominus}{\vec{\tau}}$} & disease duration & 0.217 & -0.019 & 0.004 & $<0.001$ & $-0.027--0.010$ & \\
\hline & SENS & 0.242 & -0.010 & 0.002 & $<0.001$ & $-0.015--0.006$ & \\
\hline & $\ln (E S R)$ & 0.095 & -0.159 & 0.057 & 0.007 & $-0.273--0.045$ & \\
\hline & $\ln (\sqrt{\mathrm{CRP}} \sqrt{\mathrm{CRP}})$ & 0.059 & -0.131 & 0.062 & 0.037 & $-0.254--0.008$ & \\
\hline & $\mathrm{HAQ}$ & 0.056 & -0.183 & 0.088 & 0.041 & $-0.358--0.008$ & \\
\hline & inflammation & 0.121 & -0.297 & 0.044 & 0.002 & $-0.484--0.111$ & \\
\hline & glucocorticoids & 0.059 & -0.199 & 0.093 & 0.035 & $-0.383--0.014$ & \\
\hline \multirow{5}{*}{$\underset{\downarrow}{\gtrless}$} & disease duration & 0.268 & -0.082 & 0.016 & $<0.001$ & $-0.113--0.051$ & \\
\hline & SENS & 0.279 & -0.044 & 0.008 & $<0.001$ & $-0.061--0.028$ & \\
\hline & $\mathrm{HAQ}$ & 0.059 & -0.764 & 0.356 & 0.035 & $-1.474--0.054$ & \\
\hline & inflammation & 0.073 & -0.937 & 0.389 & 0.019 & $-1.712--0.162$ & \\
\hline & CSD & 0.172 & -1.530 & 0.390 & $<0.001$ & $-2.307--0.753$ & \\
\hline \multirow{9}{*}{$\sum_{n}^{-1}$} & disease duration & 0.152 & -0.108 & 0.030 & $<0.001$ & $-0.167--0.049$ & \\
\hline & SENS & 0.169 & -0.060 & 0.015 & $<0.001$ & $-0.091--0.029$ & \\
\hline & $\ln (E S R)$ & 0.260 & -1.140 & 0.406 & 0.006 & $-1.949--0.331$ & \\
\hline & $\ln (\sqrt{\mathrm{CRF}} \sqrt{\mathrm{CRP}})$ & 0.092 & -1.173 & 0.429 & 0.008 & $-2.028--0.318$ & \\
\hline & $\sqrt{\mathrm{HAQ}}$ & 0.076 & -2.104 & 0.851 & 0.016 & $-3.799--0.408$ & \\
\hline & inflammation & 0.100 & -1.929 & 0.670 & 0.005 & $-3.264--0.594$ & \\
\hline & glucocorticoids & 0.085 & -1.690 & 0.643 & 0.010 & $-2.971--0.408$ & \\
\hline & DAS28 $8_{\mathrm{ESR}}$ activity & 0.086 & -2.033 & 0.769 & 0.010 & $-3.565--0.501$ & \\
\hline & CDAI activity & 0.052 & -1.442 & 0.720 & 0.049 & $-2.876--0.007$ & \\
\hline \multirow{6}{*}{$\stackrel{N}{\sum}$} & disease duration & 0.254 & -0.026 & 0.005 & $<0.001$ & $-0.036--0.016$ & \\
\hline & SENS & 0.274 & -0.014 & 0.003 & $<0.001$ & $-0.020--0.009$ & \\
\hline & $\sqrt{\mathrm{HAQ}}$ & 0.067 & -0.367 & 0.160 & 0.025 & $-0.686--0.048$ & \\
\hline & inflammation & 0.076 & -0.314 & 0.127 & 0.016 & $-0.567--0.061$ & \\
\hline & CSD & 0.146 & -0.461 & 0.130 & 0.001 & $-0.719--0.202$ & \\
\hline & glucocorticoids & 0.061 & -0.266 & 0.122 & 0.032 & $-0.509--0.023$ & \\
\hline \multirow{5}{*}{$\sum_{\frac{1}{K}}^{-1}$} & disease duration & 0.082 & -0.198 & 0.077 & 0.012 & $-0.351--0.045$ & \\
\hline & SENS & 0.089 & -0.108 & 0.040 & 0.009 & $-0.189--0.028$ & \\
\hline & glucocorticoids & 0.061 & -3.537 & 1.623 & 0.032 & $-6.771--0.304$ & \\
\hline & DAS28 $8_{\text {FSR }}$ activity & 0.078 & -4.818 & 1.922 & 0.014 & $-8.648--0.988$ & \\
\hline & CDAl activity & 0.052 & -3.587 & 1.792 & 0.049 & $-7.158--0.017$ & \\
\hline \multirow{4}{*}{$\underset{\frac{1}{4}}{\stackrel{N}{\Sigma}}$} & disease duration & 0.165 & -0.049 & 0.013 & $<0.001$ & $-0.074--0.023$ & \\
\hline & SENS & 0.167 & -0.026 & 0.007 & $<0.001$ & $-0.039--0.013$ & \\
\hline & CSD & 0.052 & -0.634 & 0.316 & 0.048 & $-1.262--0.005$ & \\
\hline & glucocorticoids & 0.052 & -0.567 & 0.283 & 0.049 & $-1.131--0.002$ & \\
\hline
\end{tabular}

Significant whole body lean tissue differences appear when other variables are used. Giles et al. (30) and Dao et al. (7) reported that whole body lean mass divided by square height does not differ sig- nificantly (FFMI2 in our study), but that the ratio of whole body lean and adipose content is significantly lower in RA patients (FFMI3 in our study, which we found to be non-significant, probably because our 
normal subjects had a significantly higher BMI and equivalent whole fat mass compared to the RA patients). The loss of muscle mass seems to be more pronounced in the members, as studies report in accordance with our data. Female RA patients have significantly lower appendicular lean mass than controls $(9,31)$, although there are reports in which this difference failed to reach statistical significance (30). Whether regional and selective or whether whole body and nonselective, the lean mass loss is more prevalent among the female RA population as indicated by the higher frequency of sarcopenia compared to controls $(7,30)$. Our data showed that a SMI2-derived definition of low muscle mass is more appropriate for classification purposes and that the female RA patients have a 3-fold higher risk of low muscle mass than controls, as Giles et al. also found (30).

Our data showed that RA-specific variables correlate and are significant predictors of lean mass (Tables 5 and 6). Disease duration was a strong predictor of low muscle mass, both whole body and appendicular, in female RA patients, a finding which confirms some literature results $(7,9,26,33)$, and contradicts others $(30,31)$. Since disease duration is associated with radiologic progression, disability and co-morbidity, one would have expected to observe a negative influence on lean mass in RA, the differences in reports being probably caused by the extent of disease duration and sampling methods. Similar observations were made with $\operatorname{HAQ}(7,26,30)$, inflammation markers $(8,26)$, clinical joint deformity (30), which had a significant negative effect on lean mass, while auto-antibody seropositivity and DMARD treatment produced no significant differences (9).

The points where our results differ from the literature are disease activity and glucocorticoid treatment. As with other reports $(9,30)$, the absolute values of composite disease activity scores, DAS28 namely, did not correlate nor did it predict lean mass indices, but at a nominal level analysis of our data notable results began to appear (for example patients with CDAI-remission had a lower prevalence of sarcopenia and a higher lean mass body percent than CDAI-MDA patients - Tables 2 and 4). Our glucocorticoid treated RA patients had significantly lower whole body and appendicular lean tissue indices, sarcopenia was more frequent among these patients and this status was a significant predictor of low muscle mass and lean tissue indices, while the majority of reports deny any cross-sectional effect of glucocorticoid treatment on lean body composition (7-9,31). Clinical and fundamental research has shown that exogenous glucocorticoids are associated with skeletal muscle catabolism, atrophy and wasting (34-36), therefore one would expect that long-term glucocorticoids contribute to the loss of muscle mass in the already at-risk RA patients. An explanation of this report discrepancy would be on one hand the disease duration (Book et al. studied early RA patients with disease duration below 12 months) (9), and on the other hand a less clear history of the exposure to glucocorticoid treatment (exusers, current-users or both, and never-users).

\section{Study limitations}

The cross-sectional design of the study did not allow follow-up of patients and dynamic observations of the whole body lean tissue. Data regarding diet were not included in the study design. For a thorough comparison between RA patients and controls regarding lean tissue indices, a BMI-match may have increased the objectivity of the observation. The cumulative dose of glucocorticoids and their treatment periods could not be objectively recorded.

\section{CONCLUSION}

In comparison to normal subjects, women with RA have significantly lower appendicular lean tissue DXA indices, higher prevalence of sarcopenia and lower levels of physical activity. Disease duration, inflammation, disease activity, quality of life, clinical and radiographic progression and glucocorticoid treatment are negatively correlated with lean body composition, predict whole body and appendicular lean tissue and are independent risk factors for sarcopenia and rheumatoid cachexia. The diagnosis of RA in itself is a significant predictive factor of sarcopenia.

\section{Acknowledgments}

The study was funded by the European Social Fund, contract number POSDRU/159/1.5/S/137390. The author would like to thank dr. Irina Ban for excellent technical expertise in translating the physical activity questionnaire. 


\section{REFERENCES}

1. Summers G.D., Metsios G.S., Stavropoulos-Kalinoglou A., Kitas G.D. Rheumatoid cachexia and cardiovascular disease. Nat Rev Rheumatol 2010 Aug; 6(8):445-51.

2. Hernandez-Hernandez V., Ferraz-Amaro I., az-Gonzalez F. Influence of disease activity on the physical activity of rheumatoid arthritis patients. Rheumatology (Oxford) 2014 Apr; 53(4):722-31.

3. Walsmith J., Abad L., Kehayias J., Roubenoff R. Tumor necrosis factor-alpha production is associated with less body cell mass in women with rheumatoid arthritis. J Rheumatol 2004 Jan; 31(1):23-9.

4. Summers G.D., Deighton C.M., Rennie M.J., Booth A.H. Rheumatoid cachexia: a clinical perspective. Rheumatology (Oxford) 2008 Aug; 47(8):1124-31

5. Konijn N.P., van Tuyl L.H., Bultink I.E., Lems W.F., Earthman C.P., van Bokhorst-de van der Schueren M.A. Making the invisible visible: bioelectrical impedance analysis demonstrates unfavourable body composition in rheumatoid arthritis patients in clinical practice. Scand J Rheumatol 2014; 43(4):273-8.

6. Lombard L.A., du Plessis L.M., Visser J. Body composition of rheumatoid arthritis patients in the City of Cape Town, South Africa Clin Rheumatol 2014 Apr; 33(4):467-76.

7. Dao H.H., Do Q.T., Sakamoto J. Abnormal body composition phenotypes in Vietnamese women with early rheumatoid arthritis. Rheumatology (Oxford) $2011 \mathrm{Jul}$; 50(7):1250-8.

8. Engvall I.L., Brismar K., Hafstrom I., Tengstrand B. Treatment with low-dose prednisolone is associated with altered body composition but no difference in bone mineral density in rheumatoid arthritis patients: a controlled cross-sectional study. Scand J Rheumatol 2011 May; 40(3):161-8.

9. Book C., Karlsson M.K., Akesson K., Jacobsson L.T. Early rheumatoid arthritis and body composition. Rheumatology (Oxford) 2009 Sep; 48(9):1128-32.

10. Podenphant J., Gotfredsen A., Engelhart M., Andersen V., Heitmann B.L., Kondrup J. Comparison of body composition by dual energy X-ray absorptiometry to other estimates of body composition during weight loss in obese patients with rheumatoid arthritis. Scand J Clin Lab Invest 1996 Nov; 56(7):615-25.

11. Kim J., Wang Z., Heymsfield S.B., Baumgartner R.N., Gallagher D. Total-body skeletal muscle mass: estimation by a new dualenergy X-ray absorptiometry method. Am J Clin Nutr 2002 Aug; 76(2):378-83.

12. Aletaha D., Neogi T., Silman A.J., Funovits J., Felson D.T., Bingham C.O., III, et al. 2010 Rheumatoid arthritis classification criteria: an American College of Rheumatology/European League Against Rheumatism collaborative initiative. Arthritis Rheum 2010 Sep; 62(9):2569-81.

13. Pincus T., Yazici Y., Bergman M. Development of a multidimensional health assessment questionnaire (MDHAQ) for the infrastructure of standard clinical care. Clin Exp Rheumatol 2005 Sep; 23(5 Suppl 39):S19-S28.

14. van der H.D., Dankert T., Nieman F., Rau R., Boers M. Reliability and sensitivity to change of a simplification of the Sharp/van der Heijde radiological assessment in rheumatoid arthritis. Rheumatology (Oxford) 1999 Oct; 38(10):941-7.

15. Prevoo M.L., van ,t Hof M.A., Kuper H.H., van Leeuwen M.A., van de Putte L.B., van Riel P.L. Modified disease activity scores that include twenty-eight-joint counts. Development and validation in a prospective longitudinal study of patients with rheumatoid arthritis. Arthritis Rheum 1995 Jan; 38(1):44-8.

16. Aletaha D., Nell V.P., Stamm T., Uffmann M., Pflugbeil S., Machold K., et al. Acute phase reactants add little to composite disease activity indices for rheumatoid arthritis: validation of a clinical activity score. Arthritis Res Ther 2005; 7(4):R796-R806.

17. Lohman M., Tallroth K., Kettunen J.A., Marttinen M.T. Reproducibility of dual-energy $\mathrm{x}$-ray absorptiometry total and regional body composition measurements using different scanning positions and definitions of regions. Metabolism 2009 Nov; 58(11):1663-8.

18. Cruz-Jentoft A.J., Baeyens J.P., Bauer J.M., Boirie Y., Cederholm T., Landi F., et al. Sarcopenia: European consensus on definition and diagnosis: Report of the European Working Group on Sarcopenia in Older People. Age Ageing 2010 Jul; 39(4):412-23.
19. Muscaritoli M., Anker S.D., Argiles J., Aversa Z., Bauer J.M., Biolo G., et al. Consensus definition of sarcopenia, cachexia and pre-cachexia: joint document elaborated by Special Interest Groups (SIG) "cachexia-anorexia in chronic wasting diseases" and „nutrition in geriatrics". Clin Nutr 2010 Apr; 29(2):154-9.

20. Evans W.J., Morley J.E., Argiles J., Bales C., Baracos V., Guttridge D., et al. Cachexia: a new definition. Clin Nutr 2008 Dec; 27(6):793-9.

21. Hull H.R., Thornton J., Wang J., Pierson R.N., Jr., Kaleem Z., Pi-Sunyer $\mathbf{X}$., et al. Fat-free mass index: changes and race/ethnic differences in adulthood. Int J Obes (Lond) 2011 Jan; 35(1):121-7.

22. Baumgartner R.N., Koehler K.M., Gallagher D., Romero L., Heymsfield S.B., Ross R.R., et al. Epidemiology of sarcopenia among the elderly in New Mexico. Am J Epidemiol 1998 Apr 15; 147(8):755-63.

23. Newman A.B., Kupelian V., Visser M., Simonsick E., Goodpaster B., Nevitt M., et al. Sarcopenia: alternative definitions and associations with lower extremity function. J Am Geriatr Soc 2003 Nov; 51(11):1602-9.

24. Delmonico M.J., Harris T.B., Lee J.S., Visser M., Nevitt M., Kritchevsky S.B., et al. Alternative definitions of sarcopenia, lower extremity performance, and functional impairment with aging in older men and women. J Am Geriatr Soc 2007 May; 55(5):769-74.

25. Engvall I.L., Elkan A.C., Tengstrand B., Cederholm T., Brismar K., Hafstrom I. Cachexia in rheumatoid arthritis is associated with inflammatory activity, physical disability, and low bioavailable insulin-like growth factor. Scand J Rheumatol 2008 Sep; 37(5):321-8.

26. Elkan A.C., Engvall I.L., Cederholm T., Hafstrom I. Rheumatoid cachexia, central obesity and malnutrition in patients with low-active rheumatoid arthritis: feasibility of anthropometry, Mini Nutritional Assessment and body composition techniques. Eur J Nutr 2009 Aug; 48(5):315-22.

27. Metsios G.S., Stavropoulos-Kalinoglou A., Panoulas V.F., Sandoo A. Toms T.E., Nevill A.M., et al. Rheumatoid cachexia and cardiovascular disease. Clin Exp Rheumatol 2009 Nov; 27(6):985-8.

28. Armstrong T., Bull F. Development of the World Health Organization Global Physical Activity Questionnaire (GPAQ). Journal of Public Health 2006 Jun 2; 14(2):66-70.

29. Bull F.C., Maslin T.S., Armstrong T. Global physical activity questionnaire (GPAQ): nine country reliability and validity study. J Phys Act Health 2009 Nov; 6(6):790-804.

30. Giles J.T., Ling S.M., Ferrucci L., Bartlett S.J., Andersen R.E., Towns M., et al. Abnormal body composition phenotypes in older rheumatoid arthritis patients: association with disease characteristics and pharmacotherapies. Arthritis Rheum 2008 Jun $15 ; 59(6): 807-15$.

31. Westhovens R., Nijs J., Taelman V., Dequeker J. Body composition in rheumatoid arthritis. Br J Rheumatol 1997 Apr; 36(4):444-8

32. Sahin G., Guler H., Incel N., Sezgin M., As I. Soft tissue composition, axial bone mineral density, and grip strength in postmenopausal Turkish women with early rheumatoid arthritis: Is lean body mass a predictor of bone mineral density in rheumatoid arthritis? Int J Fertil Womens Med 2006 Mar; 51(2):70-4.

33. Elkan A.C., Hakansson N., Frostegard J., Cederholm T., Hafstrom I. Rheumatoid cachexia is associated with dyslipidemia and low levels of atheroprotective natural antibodies against phosphorylcholine but not with dietary fat in patients with rheumatoid arthritis: a cross-sectional study. Arthritis Res Ther 2009; 11(2):R37.

34. Schakman O., Gilson H., Kalista S., Thissen J.P. Mechanisms of muscle atrophy induced by glucocorticoids. Horm Res 2009 Nov; 72 Suppl 1:36-41.

35. Menconi M., Fareed M., O'Neal P., Poylin V., Wei W., Hasselgren P.O. Role of glucocorticoids in the molecular regulation of muscle wasting. Crit Care Med 2007 Sep; 35(9 Suppl):S602-S608.

36. Hasselgren P.O. Glucocorticoids and muscle catabolism. Curr Opin Clin Nutr Metab Care 1999 May; 2(3):201-5. 